\title{
ORIGINAL
}

\section{IMPACTO DE LA MORBILIDAD EN LA SALUD DE LA POBLACIÓN DEL PAÍS VASCO 2002-2007: UNA VISIÓN INTEGRAL A TRAVÉS DE LAS ESPERANZAS DE SALUD (*)}

\section{Unai Martín (1), Santiago Esnaola (2), Covadonga Audicana (3) y Amaia Bacigalupe (1,2).}

(1) Departamento de Sociología 2. Universidad del País Vasco-Euskal Herriko Unibertsitatea (UPV/EHU)

(2) Servicio de Estudios e Investigación Sanitaria. Departamento de Sanidad y Consumo. Gobierno Vasco

(3) Servicio de Registros e Información Sanitaria. Departamento de Sanidad y Consumo. Gobierno Vasco

$\left(^{*}\right)$ Este trabajo ha contado para su realización con la financiación del Instituto Vasco de Innovación Sanitaria (O+Berri), así como del Departamento de Sanidad y Consumo (Ayuda para trabajos de investigación comisionada 2009/02).

No existen conflictos de interés

\section{RESUMEN}

Fundamentos: La estimación del impacto de la morbilidad en la salud de la población es esencial para la planificación sanitaria. El objetivo consistió en estimar este impacto utilizando la esperanza de vida libre de discapacidad y en analizar si la hipotética eliminación de diferentes grupos de enfermedades hubiera conllevado una compresión o expansión de la morbilidad.

Métodos: Estudio transversal referido a la población residente en la Comunidad Autónoma del País Vasco. Se combinaron datos de mortalidad (2002-2006), de la encuesta de salud del País Vasco (2007) y de población. Se estimó el impacto de los grupos de enfermedades y de enfermedades seleccionadas sobre las tasas de mortalidad, la esperanza de vida y los años potenciales de vida perdidos (APVP), y la discapacidad (número absoluto y tasas), así como de manera conjunta a través de la esperanza de vida libre de discapacidad (EVLD), calculada mediante el método Sullivan.

Resultados: Las enfermedades que provocaron un mayor impacto sobre la mortalidad en los hombres fueron los tumores $(35,2 \%$ de las muertes y el $39,3 \%$ de los APVP), y en las mujeres las enfermedades circulatorias $(34,5 \%$ del total de muertes) y los tumores $(43,6 \%$ del total de APVP). En la discapacidad las de mayor impacto fueron las enfermedades osteomusculares, que provocaron el $26,6 \%$ y el $45,2 \%$ del total de casos en hombres y mujeres. Las causas con un mayor impacto conjunto fueron las del aparato circulatorio (4,2 años de EVLD en hombres y 3,8 en mujeres). Sin embargo, las enfermedades osteomusculares fueron las que produjeron un mayor impacto sobre los años de vida con discapacidad.

Conclusiones: Las enfermedades con mayor impacto en la salud fueron las del sistema circulatorio, los tumores y las del sistema osteomuscular. La eliminación de este último grupo de enfermedades hubiera provocado una compresión de la morbilidad provocando la mayor reducción en años vividos con discapacidad entre el total de causas.

Palabras clave: Costo de enfermedad, morbilidad, mortalidad, esperanza de vida, estado de salud, España.

Correspondencia:

Unai Martín Roncero

Departamento de Sociología 2

Facultad de Ciencias Sociales y de la Comunicación

Universidad del País Vasco

48940 Leioa (Bizkaia)

unai.martin@ehu.es

\section{ABSTRACT}

Impact of Morbidity on the Health of the Basque Country Population 2002-2007: A Comprehensive Approach through Health Expectancies

Background: The estimation of the impact of morbidity on health is essential to health planning. The objective was to estimate this impact using disability free life expectancy, and to analyze whether the hypothetical elimination of various diseases would have led to a compression or expansion of morbidity.

Methods: Cross-sectional study on the population of the Basque Country. Data on mortality (2002-2006), health survey data (2007) and population based data were used. The impact of different groups of diseases on mortality rates, years of life and potential years of life lost (PYLL) and disability (absolute number and rates) were calculated. An integrated analysis was also done, using disability free life expectancy (DFLE), using the Sullivan method.

Results: The diseases causing the greatest impact on mortality were tumours among men $(35,2 \%$ and $39,3 \%$ of deaths and PYLL respectively), and circulatory diseases (34,5\% of deaths) and tumours $(43,6 \%$ of PYLL) among women. Osteomuscular diseases had a major impact on disability, causing the $26,6 \%$ and the $45,2 \%$ of the total cases in men and women). Circulatory diseases had the highest impact as a whole ( 4.2 years of DFLE in men and 3.8 in women). However, osteomuscular diseases had the highest influence on years of life with disability.

Conclusions: The diseases which caused the overall greatest impact on mortality and disability were circulatory system related ones, tumors, and osteomuscular diseases. The elimination of this last group of diseases would have led to a morbidity compression, meaning the greatest reduction in life years with disability among all the causes.

Keywords: Cost of illness. Morbidty. Mortality. Health expectancy. Health status. Spain. 


\section{INTRODUCCIÓN}

El conocimiento del impacto que las diferentes enfermedades tienen en la salud de la población constituye un aspecto esencial para la planificación sanitaria y la asignación de recursos. Tradicionalmente, este impacto se ha medido utilizando principalmente indicadores basados en la mortalidad, como los años potenciales de vida perdidos ${ }^{1-3}$. Sin embargo, en la mayor parte de las poblaciones occidentales la mortalidad es baja, se concentra en edades avanzadas y son prevalentes las enfermedades poco letales pero con un impacto importante sobre la calidad de vida relacionada con la salud. Por ello, los indicadores basados exclusivamente en la mortalidad han perdido capacidad para resumir la salud poblacional y, por lo tanto, para estimar el impacto que la morbilidad tiene en el estado de salud de las poblaciones ${ }^{4}$. Por otra parte, se han utilizado otro tipo de indicadores de uso de servicios sanitarios o variables de estado de salud, como la discapacidad, la calidad de vida relacionada con la salud o la salud percibida ${ }^{5-6}$ que, sin embargo, no permiten valorar de forma integrada los efectos no letales y letales de las diferentes enfermedades. De forma alternativa, las medidas resumen de la salud de la población ${ }^{7}$ permiten obtener un único indicador que resume ambos impactos, por lo que ya han sido utilizadas en el contexto internacional para el estudio del impacto sobre la salud de las diferentes enfermedades, lesiones o factores de riesgo $^{8}$.

Entre estas medidas es posible distinguir dos grandes aproximaciones que difieren desde el punto de vista teórico y metodológico ${ }^{9}$, lo que conlleva diferencias en los usos potenciales de sus resulta$\operatorname{dos}^{10}$. La primera está basada en estudios epidemiológicos y opiniones de personas expertas para calcular una medida de déficit de salud, los años de vida ajustados por discapacidad (disability adjusted life years) ${ }^{11}$. Esta aproximación incluye los denominados estudios de Carga de Enfermedad (Burden of Disease BoD), entre los que destaca el Estudio de Carga Global de Enfermedad (Global Burden of Disease GBD $)^{8,12,13}$. La segunda aproximación, basada principalmente en encuestas de salud, utiliza como indicador de impacto las esperanzas de salud ${ }^{9,14-18}$. Esta aproximación ha sido utilizada para evaluar los hipotéticos efectos que la eliminación de diferentes enfermedades hubieran tenido sobre la mortalidad, la discapacidad y la salud de la población ${ }^{15}$, determinando si se produce una compresión de la morbilidad ${ }^{19}$ (aumento de los años de vida y reducción de los años con discapacidad), una expansión de la morbi$\operatorname{lidad}^{20}$ (aumento de los años de vida a costa de un incremento de los años con discapacidad) o modelos intermedios ${ }^{21}$.

En el caso de España son escasos los estudios que han tratado de estimar de forma integrada el impacto de las diferentes enfermedades sobre la salud de la población y todos ellos han sido realizados desde la primera aproximación. La mayor parte de los mismos han estimado la carga de enfermedad asociada a patologías con$\operatorname{cretas}^{22-28}$, y pocos han estudiado de manera conjunta el impacto del conjunto de la morbilidad ${ }^{29-32}$. Además, gran parte de ellos ha estimado los datos de salud para España a partir de datos sobre el estado de salud de regiones más amplias como la europea.

El objetivo de este trabajo fue calcular el impacto de los grupos de enfermedades más prevalentes en la salud de la población de la Comunidad Autónoma del País Vasco (CAPV) durante el período 20022007 utilizando como medida principal un tipo de esperanza de salud: la esperanza de vida libre de discapacidad (EVLD). Asimismo, para cada uno de esos grupos de enfermedades, se analizó si su elimina- 
ción hubiera conllevado una compresión o expansión de la morbilidad.

\section{SUJETOS Y METODOS}

\section{Diseño, población de estudio y fuentes} de información. Estudio transversal referido a la población residente en CAPV en el período 2002-2007. Se estudió el impacto de los grandes grupos de enfermedades y de algunas enfermedades concretas sobre la mortalidad y la discapacidad de manera separada y sobre ambas de forma integrada. Las enfermedades se seleccionaron por su alta prevalencia y/o impacto sobre la mortalidad (tabla 1).

Se combinaron los datos de mortalidad del período 2002-2006 con los de la Encuesta de Salud de la CAPV 2007 (ESCAV'07 ${ }^{33}$ ) y los datos de población de 2004. Los datos de mortalidad se obtuvieron de la Estadística de Defunciones de la CAPV realizada por el Instituto Vasco de Estadística (Eustat) en colaboración con el INE (datos facilitados por la Dirección de Gestión del Conocimiento y Evaluación. Departamento de Sanidad y Consumo. Gobierno Vasco). Se utilizó el número total de muertes durante el período 2002-2006 (94.540) de las que 49.834 fueron en hombres y 44.706 en mujeres. Los datos de población por edad y sexo se estimaron por interpolación lineal a partir de los datos del Censo de Población y Viviendas de 2001, y la Estadística de Población de 2006 llevada a cabo por Eustat ambos datos facilitados por el Eustat-Instituto Vasco de Estadística). Los datos de salud se obtuvieron de la Encuesta de Salud de la Comunidad Autónoma del País Vasco $2007^{33}$, dirigida a una muestra representativa (13.555 sujetos) de la población no institucionalizada de la CAPV, cuya metodología detallada ha sido publicada con anterioridad $^{33}$.

Análisis estadístico. El impacto sobre la mortalidad se valoró mediante el número de defunciones, las tasas de mortalidad (calcu- ladas para todo el periodo y expresadas de forma anual) y los años potenciales de vida perdidos totales y por grupos de enfermedades. En el caso de los años potenciales de vida perdidos se tuvo en cuenta las defunciones de menores de 1 año y se utilizó la esperanza de vida como umbral para considerar una muerte como prematura, considerando 77 años para hombres y 84 para mujeres. En el caso de la discapacidad se calculó el número total de personas con discapacidad y las tasas brutas de discapacidad totales y por grandes grupos de enfermedades y causas seleccionadas. Para el cálculo de la esperanza de vida (EV) se utilizó la tabla de vida abreviada siguiendo el método de Chiang $^{34}$, y la EVLD se calculó integrando la información de la discapacidad en la tabla de vida mediante el método de Sullivan ${ }^{35}$. Se estimó la EV, la EVLD, la esperanza de vida con discapacidad (EVD) y el porcentaje de vida que se espera vivir sin discapacidad antes y después de la hipotética eliminación de cada una de las diferentes causas o grupos de ellas. Se utilizaron tablas con "causas eliminadas" siguiendo la metodología de tablas con decrementos simples asociados, recogida en Preston et al ${ }^{17,36}$, utilizando el método propuesto por Chiang ${ }^{37}$ y asumiendo independencia entre las causas de muerte y de discapacidad. Para el cálculo de la porción de años vividos (ax) se realizaron las transformaciones tal y como se propone en Preston et $\mathrm{al}^{36}$ a partir de las propuestas de Chiang ${ }^{34}$ para las tablas de vida con todas las causas, ya que éstas fueron las que se utilizaron en el cálculo de la esperanza de vida general. La EVLD por causas eliminadas se estimó calculando las prevalencias de discapacidad una vez se eliminaron las discapacidades provocadas por la causa a eliminar. El impacto se midió tanto de manera absoluta como relativa.

Atribución de las causas de mortalidad y discapacidad a las diferentes causas. Las defunciones fueron atribuidas a cada una de las causas y grupos de enfermedad utilizando la causa básica de defunción del 
Tabla 1

Grupos de enfermedades y causas seleccionadas

\begin{tabular}{|l|l|l|}
\hline \multicolumn{1}{|c|}{ Grupo de enfermedad } & Código CIE-10 & \multicolumn{1}{|c|}{ CIE-9MC } \\
\hline Tumores & CO0-D48 & $140-239,273.1 .3,289.8$ \\
\hline Cáncer de mama & C50 & 174,175 \\
\hline Cáncer de pulmón & C33-C34 & 162 \\
\hline Enfermedades endocrinas, nutricionales y metabólicas & E00-E90 & $240-278,330.0 .1$ (excepto 273.0.1.2.3, 274) \\
\hline Diabetes mellitus & E10-E14 & 250 \\
\hline Trastornos mentales y del comportamiento & F00-F99 & $290-319$ \\
\hline Enfermedades del sistema nervioso & G00-H95 & $320-389,435$ (excepto 330.0.1) \\
\hline Enfermedades del sistema circulatorio & I00-I99 & $390-459,($ excepto 427.5, 435, 446, 459.0) \\
\hline Cardiopatía isquémica & I20-I25 & $410-414$ \\
\hline Enfermedades cerebrovasculares & I60-I69 & $430-434,436-438$ \\
\hline Enfermedades del sistema respiratorio & J00-J99 & $460-519,786.0$ \\
\hline EPOC & J40-J44, J47 & $490-492,494,496$ \\
\hline Asma & J45, J46 & 493 \\
\hline Enfermedades del sistema digestivo & K00-K93 & $520-579$ \\
\hline Enfermedades del sistema osteomuscular y del tejido conjuntivo & M00-M99 & $274,446,710-739$ \\
\hline Enfermedades del sistema genitourinario & N00-N99 & $580-629$ \\
\hline
\end{tabular}

Fuente: Registros de mortalidad de las Comunidades Autónomas e $\mathrm{INE}^{42}$

Tabla 2

Atribución del escenario de compresión o expansión de la morbilidad a partir de los cambios en la esperanza de vida (EV), la esperanza de vida libre de discapacidad (EVLD), esperanza de vida con discapacidad (EVD) y porcentaje de esperanza de vida que se espera vivir libro de discapacidad (\%EVLD/EV).

\begin{tabular}{|c|c|c|c|c|}
\hline EV & EVLD & EVD & $\%$ EVLD/EV & Escenario \\
\hline Aumenta & Aumenta & Disminuye & Aumenta & Compresión morbilidad \\
\hline Aumenta & Aumenta & Aumenta & Aumenta & Relativa compresión morbilidad \\
\hline Aumenta & Aumenta & Aumenta & Disminuye & Relativa expansión morbilidad \\
\hline Aumenta & Disminuye & Aumenta & Disminuye & Expansión de la morbilidad \\
\hline
\end{tabular}

Fuente: Elaboración propia a partir de Nusselder et al. ${ }^{15}$

Boletín Estadístico de Defunción, que en el caso del País Vasco es codificado por el Departamento de Sanidad y Consumo, utilizando la CIE-10 $0^{38}$ (tabla 1). En cuanto a la atribución de la discapacidad, además de la existencia o no de la limitación para las actividades de la vida cotidiana, en la ESCAV se recoge el motivo que originó esta limitación con una pregunta abierta que permite detallar hasta tres causas que posteriormente son codificadas por personal sanitario, utilizando la CIE-9 MC $^{39}$ (tabla 1). Un mayor detalle de la metodología de la ESCAV para estas variables así como el cuestionario se puede consultar en la publi- cación oficial de dicha encuesta ${ }^{33}$. Para calcular las tasas de discapacidad por causas específicas se consideraron todas las causas señaladas como origen de la misma. Sin embargo, en el caso de la EVLD sólo se "eliminaron" los estados de discapacidad cuando se señaló una única causa, ya que se consideró que si una persona señalaba dos causas como el origen de su discapacidad, ésta no desaparecería si se eliminara una de las causas señaladas.

Atribución del escenario de compresión o expansión de la morbilidad a la eliminación de una enfermedad. El análisis 
del impacto de la eliminación de cada causa en la compresión o expansión de la morbilidad, partiendo de lo propuesto por Nusselder et $\mathrm{al}^{15}$ se llevó a cabo teniendo en cuenta su efecto combinado sobre la EV, la EVLD, la EVD y el porcentaje de vida que se espera vivir sin discapacidad, tal y como se muestra en la tabla 2.

\section{RESULTADOS}

Impacto sobre la mortalidad. En el caso de la mortalidad masculina fueron los tumores los que provocaron un mayor número de muertes, un total de 17.522 (32,5\% del total), así como de APVP, un total de 154.883 (39,3\% del total), seguidos de las enfermedades del sistema circulatorio con 13.735 muertes $(27,6 \%$ del total) y 73.057 APVP (18,1\% del total). En el caso de las mujeres, las enfermedades del sistema circulatorio fueron las causantes del mayor impacto sobre la mortalidad por el número de muertes: 15.328 (34,3\% del total), y los tumores por el número de años potenciales de vida perdidos: 127.111 (43,6\% del total). Entre las enfermedades concretas seleccionadas, en los hombres la causa con un mayor impacto fue la cardiopatía isquémica si se considera la tasa de mortalidad, con un total de 4.649 (9,3\% del total) y el cáncer de pulmón si se atiende a los APVP, con 42.682 años (10,8\% del total). Respecto a la mortalidad total en las mujeres, las de mayor impacto fueron la enfermedad cerebrovascular con 4.615 defunciones $(10,3 \%$ del total) y la cardiopatía isquémica con 3.115 (7,0\% del total). En relación a la mortalidad prematura, destacaron el cáncer de mama: 23.720 APVP (8,1\%), seguido de la enfermedad cerebrovascular: 16.669 APVP $(5,7 \%$ del total $)$.

Impacto sobre la discapacidad. El grupo de las enfermedades del sistema osteomuscular fue el causante del mayor número de personas discapacitadas, tanto en hombres, 22.831 (26,6\%) como en mujeres, 41.811 $(45,2 \%)$. A este grupo le siguieron las enfer- medades del sistema circulatorio y las enfermedades del sistema nervioso en hombres y las enfermedades del sistema nervioso y los trastornos mentales en mujeres. Entre las enfermedades concretas, fueron la cardiopatía isquémica en hombres, $6.767(7,9 \%)$ y el tumor de mama en mujeres, $2.426(2,6 \%)$, las que provocaron una mayor discapacidad.

Impacto conjunto sobre la mortalidaddiscapacidad. Los grupos de enfermedades con mayor impacto sobre la EVLD fueron las enfermedades del sistema circulatorio $(4,2$ años en hombres y 3,8 en mujeres), los tumores (4,1 y 2,7 años), las enfermedades del sistema osteomuscular (1,3 y 2,5 años) y las enfermedades del sistema nervioso ( 1,1 y 1,6 años). Teniendo en cuenta de manera conjunta el impacto que su eliminación hubiera tenido en la EV, la EVLD, la EVD y el porcentaje que la EVLD representa sobre el total de la EV (tablas 3 y 4), se pudieron establecer diferentes grupos de enfermedades. La eliminación de las enfermedades del sistema osteomuscular, del sistema nervioso, los trastornos mentales y las enfermedades del sistema circulatorio en el caso de los hombres (sobre todo la cardiopatía isquémica) hubieran provocado un aumento en la esperanza de vida y en la EVLD (hombres: 1,3; 1,1; 0,9; 4,2 años y mujeres: 2,$5 ; 1,6: 1,1 ; 3,8$ años respectivamente), así como una disminución de la EVD (hombres; 1,$3 ; 0,6 ; 0,6 ; 0,2$, años y mujeres: 2,$3 ; 0,9 ; 0,4 ; 1,4$ años respectivamente). La eliminación de los tumores (especialmente del cáncer de pulmón en los hombres), las enfermedades del sistema circulatorio en las mujeres, así como en menor medida las de los sistemas respiratorio, digestivo y genitourinario en ambos sexos, hubieran provocado un aumento de la esperanza de vida y la EVLD (tablas 3 y 4). Sin embargo, esta hipotética eliminación también hubiera incrementado el número de años que se espera vivir con discapacidad, aumentando también el porcentaje que tales años representan respecto a la esperanza de vida total. La eliminación de las enfermedades endocrinas y las enfermedades cerebrovasculares en hombres hubiera con- 


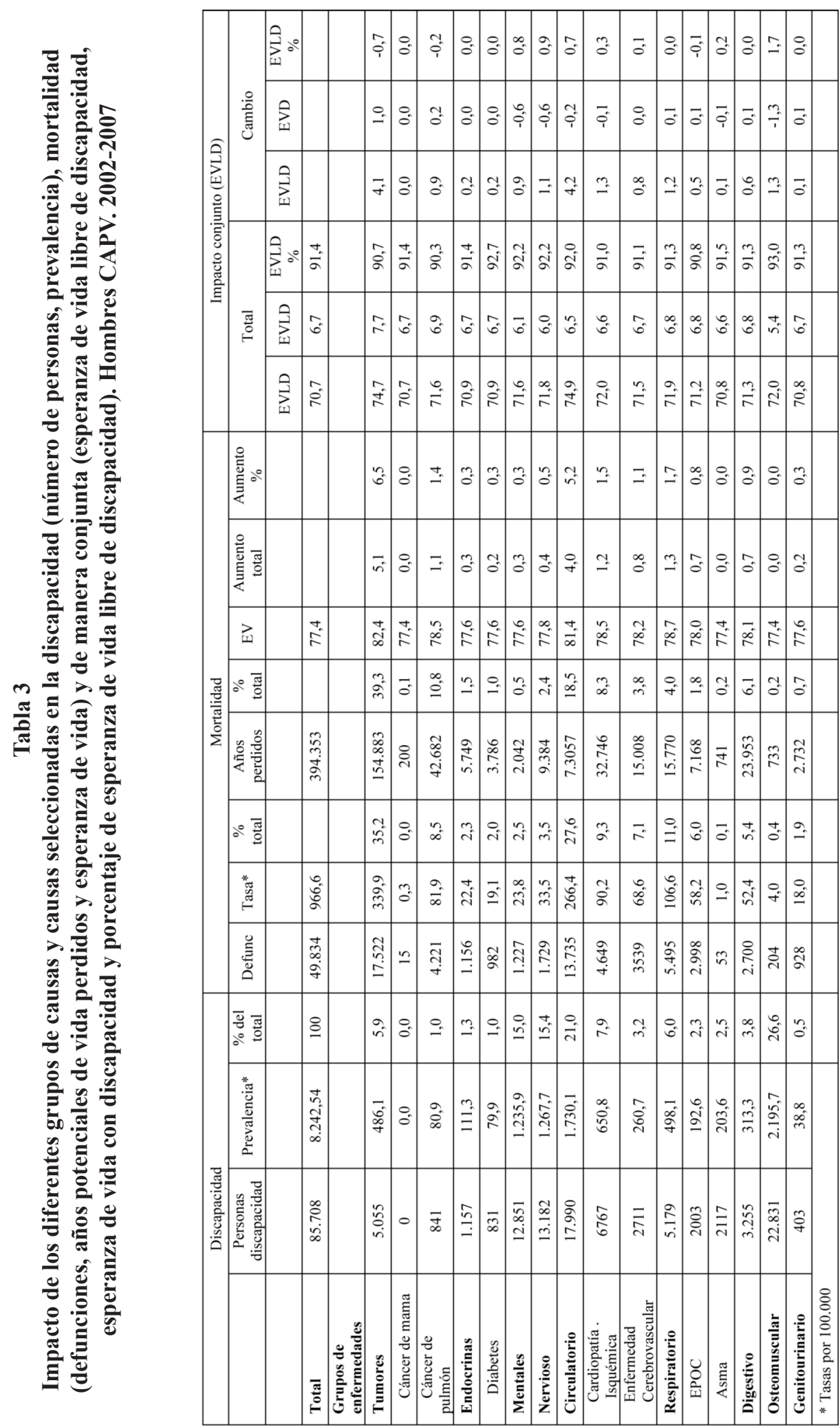




\begin{tabular}{|c|c|c|c|c|c|c|c|c|c|c|c|c|c|c|c|c|c|c|c|c|c|}
\hline ت & & & $\approx 9$ & & & $\stackrel{\vartheta}{\because}$ & $\overrightarrow{=}$ & $\cong$ & $\because$ & 8 & $\cong$ & $\Rightarrow$ & $\because$ & $\vec{s}$ & $\vec{\beta}$ & $\vec{\sigma}$ & $\therefore=$ & $\overrightarrow{9}$ & $\infty$ & $\overrightarrow{9}$ & \\
\hline 党 & & 量 & 厡 & & & 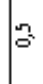 & $\cong$ & $\cong$ & $\cong$ & 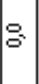 & 范 & : & 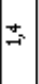 & $\cong$ & 3 & $\cong$ & $\vec{\Xi}$ & $\vec{B}$ & $\vec{w}$ & $\Rightarrow$ & \\
\hline 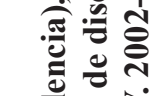 & 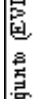 & & 念 & & & $\hat{\mathrm{s}}$ & $\stackrel{3}{\circ}$ & 3 & 志 & 3 & $\Rightarrow$ & 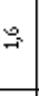 & \begin{tabular}{|l|}
$\infty$ \\
$m_{2}$
\end{tabular} & $\cong$ & $\stackrel{\infty}{\circ}$ & $\stackrel{\infty}{\circ}$ & $\cong$ & 5 & $\sqrt{2}$ & $\cong$ & \\
\hline 尝 & $\mid$ & & 昜 & $\because$ & & $\vec{*}$ & $\cong$ & $\stackrel{\circ}{\circ}$ & $\cong$ & $\stackrel{\circ}{\circ}$ & $\vec{E}$ & $\approx$ & 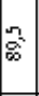 & $\stackrel{\circ}{2}$ & 蓠 & $\vec{z}$ & 2 & $=2$ & 2 & 2 & \\
\hline 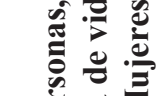 & & 恶 & 展 & $\infty$ & & 芯 & 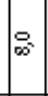 & 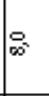 & 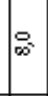 & 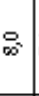 & $\approx$ & $\therefore$ & : & $\stackrel{\infty}{\infty}$ & $\infty$ & $\infty:$ & $\infty$ & $\overrightarrow{0} \vec{\infty}$ & 5 & $-\vec{\infty}$ & \\
\hline 를 & & & 晨 & $\approx$ & & 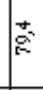 & $\approx$ & $\approx$ & $\vec{\infty}$ & $\approx$ & $\approx$ & 2 & 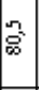 & $\approx$ & $\mathbb{2}$ & $\underset{2}{2}$ & $\Leftrightarrow$ & 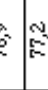 & $\approx$ & $\Leftrightarrow$ & \\
\hline 总 总 & & 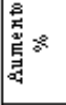 & & & & $\vec{r}$ & $\cong$ & 3 & $\cong$ & 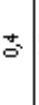 & $\approx$ & $\stackrel{\infty}{\circ}$ & $\approx$ & $\Rightarrow$ & $\exists$ & $\cong$ & $3=$ & $=$ & $\cong$ & 3 & \\
\hline 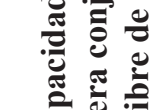 & & 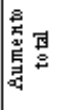 & & & & $\vec{r}$ & $\cong$ & 3 & 志 & 3 & $\because$ & 5 & $\sqrt{\infty}$ & : & 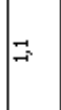 & $\Rightarrow$ & $3=$ & $5:$ & $\cong$ & $\cong$ & \\
\hline & & 售 & & 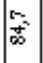 & & $\overbrace{\infty}^{\infty}$ & $\mathbb{\infty}$ & 焉 & $\vec{\infty}$ & $\begin{array}{l}0 \\
\mathbb{2}\end{array}$ & $\vec{\infty}$ & 蔡 & 齐 & 䓌 & {$\left[\begin{array}{l}\infty \\
\aleph^{\infty} \\
\infty\end{array}\right.$} & 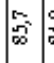 & $\begin{array}{l}0 \\
+\infty \\
\infty\end{array}$ & $\mathbb{0}$ & 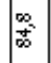 & 产 & \\
\hline 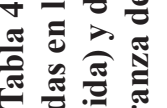 & 唄 & $\begin{array}{l}\text { 露 } \\
2 \\
2\end{array}$ & & & & 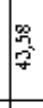 & $\vec{\infty}$ & 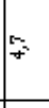 & $\vec{n}$ & $\stackrel{\infty}{\sim}$ & 索 & $\approx$ & $\overrightarrow{\mid \overrightarrow{0}}$ & 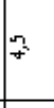 & $\sqrt{3}$ & : & $\Rightarrow$ & $=\underset{+}{\alpha}$ & $\mathbb{N}$ & $\mathbb{N}$ & \\
\hline 冚 & $E$ & 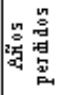 & & 导 & & $\underset{ت}{\mathbb{N}}$ & 路 & 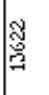 & $\mathbb{2}$ & 管 & 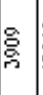 & 茧 & 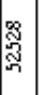 & 商 & 鹿 & & 疍 & 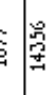 & $\stackrel{\sim}{*}$ & 刻 & \\
\hline ల్ల & & $\approx \stackrel{7}{8}$ & & & & 菏 & $\approx$ & $\because$ & $\vec{m}$ & $\stackrel{8}{r}$ & $\therefore$ & $\Rightarrow$ & 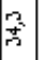 & $\approx$ & 3 & $\therefore$ & $\vec{\sim}$ & + & 3 & אִ & \\
\hline 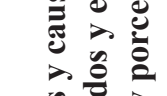 & & 福 & & 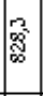 & & 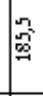 & $\because$ & $\approx$ & 芯 & సิ. & $\approx$ & 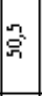 & 芧 & & $\infty$ & 忩 & 焉 & $\frac{a}{9}$ & $\stackrel{m}{=}$ & $\underset{\infty}{\infty}$ & \\
\hline 焉 & & 岂 & & 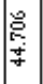 & & 莫 & 受 & 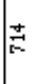 & 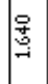 & $\overrightarrow{7}$ & 尊 & ה & 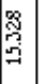 & $\nexists$ & $\vec{F}$ & 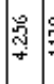 & 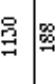 & \& & 壳 & 总 & \\
\hline 气 & & $\overrightarrow{3}$ & & & & $\therefore$ & 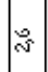 & $\cong$ & 芯 & $\stackrel{\infty}{\rightarrow}$ & 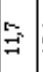 & 荵 & 芯 & $\because$ & 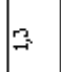 & $\because$ & $\stackrel{N}{\circ} \vec{N}$ & $\vec{s}$ & 10 & 3 & \\
\hline 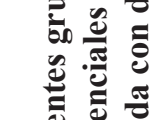 & & 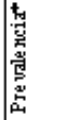 & & 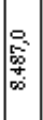 & & 党 & :ี & 3 & $\overrightarrow{2}$ & $\begin{array}{l}\text { 总 } \\
\vdots \\
\end{array}$ & $\frac{2}{2}$ & 恋 & 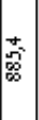 & 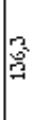 & 管 & $\stackrel{2}{*}$ & $\stackrel{8}{=}$ & $\overrightarrow{8}$ & 站 & 2 & \\
\hline 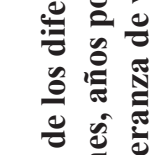 & 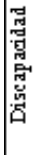 & 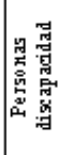 & & 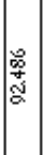 & & 总 & 蛋 & 罢 & $\frac{\$}{2}$ & : & 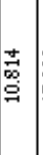 & : & 兽 & 怘 & 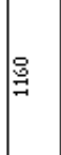 & 旅 & $\approx$ & $\int_{-i}$ & $\vec{\nabla}$ & \% & \\
\hline 冚 & & & & 氙 & 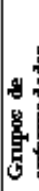 & 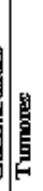 & 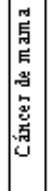 & 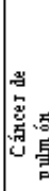 & 管 & 器 & 密 & 噌 & . & 热 & 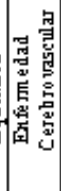 & 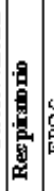 & 递 & 案 & 量 & 覞 & 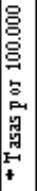 \\
\hline
\end{tabular}


llevado un aumento de la EVD, si bien el porcentaje que estos años representan en la esperanza de vida total hubiera disminuido.

\section{DISCUSIÓN}

Teniendo en cuenta el efecto conjunto sobre la mortalidad y la discapacidad, los grupos de enfermedades de mayor impacto fueron las enfermedades del sistema circulatorio, los tumores, las enfermedades del sistema osteomuscular y las enfermedades del sistema nervioso y trastornos mentales. El impacto sobre la salud de la población vasca derivado de la hipotética eliminación de los grupos de enfermedades no sólo fue diferente en términos cuantitativos sino también cualitativos. Así, la eliminación de las enfermedades del sistema osteomuscular, del sistema nervioso y los trastornos mentales, así como las del sistema circulatorio en el caso de los hombres, hubiera provocado una compresión de la morbilidad, es decir, un aumento de los años de vida acompañado de una reducción de los años con discapacidad. Sin embargo, la eliminación de los tumores y las enfermedades del sistema circulatorio en las mujeres, así como las de los sistemas respiratorio y digestivo, hubieran provocado una relativa expansión de la morbilidad, es decir, el aumento de los años de vida acompañados de un incremento paralelo de los años de vida en discapacidad y de un descenso del porcentaje que la EVLD representa en la EV. En un escenario intermedio se sitúa la eliminación de las enfermedades endocrinas que hubieran provocado una relativa compresión de la morbilidad, es decir, un aumento del número de años de vida totales y con discapacidad, pero un descenso en el porcentaje que estos representan respecto al total de esperanza de vida.

No se ha encontrado otra estimación del impacto global de los diferentes grupos de enfermedad utilizando datos de la CAPV. En el caso del Estado español, algunos estudios han estimado este impacto para el con- junto de enfermedades utilizando medidas de déficits de salud, es decir, la primera de las aproximaciones mencionada en la introducción ${ }^{29-31}$, pero no se ha encontrado ninguno que lo haga utilizando esperanzas de salud. En el ámbito internacional, la variabilidad en la metodología de los estudios no permite una comparación directa con nuestros resultados. Sin embargo, en términos generales los resultados coinciden con los descritos en otros estudios ${ }^{9,15}$. La metodología más parecida es la utilizada por Bronnum-Hansen et al. para los mayores de 65 años en Dinamarca ${ }^{17}$ : aunque los resultados derivados de la comparación deban realizarse con cautela, en base a los datos para los mayores de 65 años de la CAPV (no mostrados) parece que los tumores tienen un mayor impacto sobre los hombres en la CAPV que en Dinamarca, y la cardiopatía isquémica un impacto menor. En el caso de las mujeres mayores de la CAPV, el impacto de las enfermedades del sistema circulatorio fue mayor, sobre todo en las enfermedades cerebrovasculares, mientras que la cardiopatía isquémica tuvo un impacto menor. Las enfermedades del sistema osteomuscular también tuvieron un impacto menor en el caso de las mujeres de la CAPV.

Entre las limitaciones de este estudio, conviene tener en cuenta el posible sesgo introducido por la comorbilidad, tanto en el caso de la mortalidad (al considerar una única causa como la responsable de la muerte) como de la discapacidad. Asimismo, se usaron distintas clasificaciones para unir los datos de mortalidad (CIE-10) y discapacidad (CIE-9MC). Otra de las limitaciones se deriva del uso del método Sullivan para el cálculo de las esperanzas de salud. Este método utiliza datos de prevalencia para los estados de salud y de incidencia para la mortalidad, por lo que en el caso de la discapacidad, se tienen en cuenta efectos de enfermedades que pueden haberse producido en épocas anteriores al estudio. Sin embargo, la relativa simplicidad de los datos requeridos para su cálculo hace de éste el método 
más usado y el único posible en nuestro contexto. Por último, la ESCAV, al igual que la mayoría de encuestas de salud poblacionales, no recoge información para la población institucionalizada, lo que puede suponer un sesgo, sobre todo en la población mayor y en el caso de que alguna enfermedad aumente las probabilidades de institucionalización. Además, la manera de medir la discapacidad y sus causas en la ESCAV puede introducir sesgos clásicos de los estudios poblacionales por encuesta como la dificultad de recuerdo u otros derivados de la medida autodeclarada de la discapacidad.

Este estudio ha utilizado esperanzas de salud, lo que supone una serie de ventajas respecto a otros que utilizan otras medidas globales, como los años de vida ajustados por discapacidad. Además de la menor exigencia de datos para su cálculo, las esperanzas de salud tienen una mejor capacidad para reflejar los cambios en el impacto de las enfermedades sobre la salud ${ }^{9}$, y permiten un mejor tratamiento de la comorbilidad y la introducción de otras variables en el estudio, como las características socioeconómicas $^{16}$. Otra de las ventajas del estudio es contar con la información necesaria para estimar el impacto en la salud de las diferentes enfermedades a partir de datos de la población objeto de estudio. Ello implicó que no fuera necesario utilizar estimaciones realizadas en otras poblaciones más generales, como ocurre en algunos de los estudios que utilizan la metodología de los años de vida ajustados por discapacidad ${ }^{29-31}$. Además, la atribución de los estados de discapacidad a las causas se realizó de una manera directa y no a partir de la relación entre el estado de salud de la persona y las enfermedades crónicas que presenta?

Los resultados de este estudio mejoran el conocimiento actual del impacto de las diferentes enfermedades sobre la salud de la población siendo de gran valor para las políticas de salud. Los resultados relativos al impacto de las diferentes enfermedades medidos a través de la esperanza de vida libre de discapacidad difieren del patrón mostrado según las medidas de mortalidad, lo que tiene importantes implicaciones desde el punto de vista de asignación de recursos y priorización de servicios. Considerar únicamente la mortalidad llevará, por ejemplo, a infraestimar la importancia de enfermedades crónicas como las enfermedades del sistema osteomuscular, las enfermedades del sistema nervioso o los trastornos mentales. Es de gran relevancia la introducción de este tipo de análisis en la monitorización del estado de salud de las poblaciones con el objetivo de ayudar a una planificación y asignación de recursos que refleje más adecuadamente la influencia que las diferentes enfermedades tienen en la salud de la población.

Resulta necesario seguir avanzando en esta línea de investigación incorporando además mejoras conceptuales y metodológicas. Estas mejoras deberán incluir la estimación del impacto de las enfermedades siguiendo otras metodologías alternativas (Global Burden of Disease) ${ }^{8}$ así como mejoras en la metodología empleada. Asimismo, futuros estudios deberían explorar en nuestro contexto la posibilidad de introducir en estas estimaciones, además de otras enfermedades, factores de riesgo como el tabaco, el sedentarismo o la desigualdad social. Por último, se debería tener en cuenta si el impacto de estas enfermedades o factores de riesgo es igual en los diferentes grupos sociales y cuál es la contribución de las diferentes enfermedades y factores de riesgo sobre las desigualdades sociales de la salud, tal y como se ha realizado en otros contextos ${ }^{40,41}$.

\section{BIBLIOGRAFÍA}

1. Centers for Disease Control. Premature mortality in the United States: public health issues in the use of years of potential life lost. MMWR. 1986;35:1-11S. 
2. Romeder JM, McWhinnie JR. Potential years of life lost between ages 1 and 70: an indicator of premature mortality for health planning. Int $\mathrm{J}$ Epidemiol. 1977; 6:143-151.

3. Lapostolle A, Lefranc A, Gremy I, Spira A. Premature mortality measure: comparison of deaths before 65 and expected years of life lost. Rev Epidemiol Sante Publique. 2008; 56:245-252.

4. Schopper D, Torres AM, Pereira J, Ammon C, Ceunde N, Alonso $\mathrm{M}$ et al. Setting health priorities in a Swiss canton: what do different methods tell us? J Epidemiol Community Health. 2000; 54:388-393.

5. Perruccio AV, Power JD, Badley EM. The relative impact of 13 chronic conditions across three different outcomes. J Epidemiol Community Health. 2007; 61:1056-1061.

6. Van Den Bos GAM. The burden of chronic diseases in terms of disability, use of health care and healthy life expectancies. Eur J Public Health. 1995; 5(1):29-34.

7. Murray CJL, Salomon JA, Mathers CD, Lopez AD, editors. Summary measures of population health. Concepts, ethics, measurements and applications. Geneva: World Health Organization; 2002.

8. Murray CJL, Lopez AD, editors. The global burden of disease: a comprehensive assessment of mortality and disability from diseases, injuries and risk factors in 1990 and projected to 2020. Global Burden of Disease and Injury, Vol 1. Cambridge: Harvard School of Public Health on behalf of WHO; 1996.

9. Manuel DG, Schultz SE, Kopec JA. Measuring the health burden of chronic disease and injury using health adjusted life expectancy and the Health Utilities Index. J Epidemiol Community Health. 2002; 56:843850 .

10. Mathers CD. Cause-deleted health expectancies. En: Robine JM, Jagger C, Mathers CD, Crimmins EM, Suzman RM, editors. Determining Health Expectancies. Wiley, West Sussex; 2002.p.149-174.

11. Murray CJL, Lopez A. Quantifying disability: data, methods and results. Bull World Health Organ. 1994; 72:481-94.

12. World Health Organization. World Health Report 2000. Health Systems: improving Performance. Geneva: WHO; 2000.

13. World Health Organization. The global burden of disease: 2004 update. Geneva: WHO; 2008.
14. Colvez A, Blanchet M. Potential gains in life expectancy free of disability: a tool for health planning. Int J Epidemiol. 1983; 12(2):224-229.

15. Nusselder WJ, van der Velden K, Sonsbeek JLA, Lenior ME, van der Bos GA. The elimination of selected chronic diseases in a population: the compression and expansion of morbidity. Am J Public Health. 1996; 86(2):187-193.

16. Manuel DG, Luo W, Ugnat AM, Mao Y. Causesdeleted health-adjusted life expectancy of Canadians with selected chronic conditions. Chronic Diseases in Canada. 2003; 24(4):108-115.

17. Bronnum-Hansen H, Juel K, Davidsen M. The burden of selected diseases among older people in Denmark. J Aging Health. 2006; 18(4): 491-506.

18. Pérès K, Jagger C, Matthews FE. Impact of late-life self-reported emotional problems on disability-free life expectancy: results from the MRC Cognitive Function and Ageing Study. Int J Geriatr Psychiatry. 2008; 23(6):643-9.

19. Fries JF. Aging, natural death, and the compression of morbidity. New England Journal of Medicine. 1980; 303:130-135

20. Gruenberg EM. The failure of success. Milbank Memorial Fund Quarterly/Health and Society. 1977; $55: 3-24$

21. Manton KG. A Changing concepts of morbidity and mortality in the elderly population. Milbank Memorial Fund Quarterly/Health and Society.1982; 60:183-244.

22. Cortés M, Pereira J, Peña-Rey I, Génova R, Amela C. Carga de enfermedad de la población española de 15 años para el año 1999. Med Clin (Barc). 2004; 122(10):369-371.

23. Bermúdez-Tamayo C, Martín Martín JJ, RuizPerez I, Olry de Labry Lima A. Factors associated with improvement in disability-adjusted life years in patients with HIV/AIDS. BMC Public Health. 2008; $8: 362$

24. Sánchez-Valle E, Posada M, Villaverde-Hueso A, Touriño E, Ferrari-Arroyo MJ, Boada L et al. Estimating the Burden of Disease for Autism Spectrum Disorders in Spain in 2003. J Autism Dev Disord. 2008; 38(2):288-296.

25. Cortés M, Pereira J, Peña-Rey I, Génova R, Amela C. Carga de enfermedad atribuible a las afecciones inmunoprevisibles en la población infanto-juvenil española. Gac Sanit. 2004; 18(4):312-320. 
26. Villaverde-Hueso A, Sánchez-Valle E, Alvarez E, Morant C, Carreira PE, Martín-Arribas MC et al. Estimating the burden of sleroderma disease in Spain. J Rheumatol. 2007; 34(11):2236-42.

27. Ayuso-Mateos JL, Gutierrez P, Haro JM, Salvador L, Vázquez FJ, Negrín MA et al. Reducing the Burden of Mental Illness in Spain. Bilbao: Fundación BBVA; 2008.

28.Cubo E, Alvarez E, Morant C, De Pedro J, Martínez $\mathrm{P}$, Genova R et al. Burden of disease related to Parkinson's disease in Spain in the year 2000. Mov Disord. 2005; 20:1481-7.

29. Gènova R, Álvarez E, Morant G. Estimación de la carga de enfermedad en España en el año 2000. Cuadernos Geográficos. 2005; 36:571-6.

30. IESPCM. Informe del estado de salud de la población de la Comunidad de Madrid 2006. Madrid: Instituto de salud Pública, Dirección General de Salud Pública y Alimentación, Consejería de Sanidad y Consumo; 2008.

31. Consellería de Sanidade. Carga de enfermedad en Galicia. Disponible en: www.qaliciasalud.netllnvestiqacion/Docs/lnformeAVADs.pdf

32. Catalá F, Álvarez E, Gènova R, Ginestar M. Relación en España entre la investigación sanitaria financiada por el Sistema Nacional de Salud y la carga de enfermedad en la comunidad. Rev Esp Salud Publica. 2009; 83:137-151.

33. Pérez Y. Esnaola S. Ruiz R. Diego M. Aldasoro E. Calvo M. Bacigalupe A. llano J. Martín U. Encuesta de Salud de la C.A del País Vasco 2007. Vitoria-Gasteiz: Servicio Central de Publicaciones del Gobierno Vasco; 2010

34. Chiang CL. The life table and its applications. Florida: Robert E. Krieger Publ. Co.; 1984.

35. Sullivan DF. A single index of mortality and morbidity. HSMHA Health Rep. 1971; 86:347-54.

36. Preston SH, Heuveline P, Guillot M. Demography: measuring and modelling population processes. Oxford: Blackwell; 2003.

37. Chiang CL. Introduction to stochastic processes in biostatistics. New York: John Wiley; 1968.

38. Organización Panamericana de la Salud. Clasificación Estadística Internacional de Enfermedades y Problemas relacionados con la Salud. Décima revisión. Volumen 1. Washington DC: OPS; 2008.
39. CIE-9-MC Clasificación Internacional de Enfermedades, $9^{\mathrm{a}}$ Revisión Modificación Clínica, $6^{\mathrm{a}}$ Edición, Enero 2008 .

40. Nusselder WJ, Loman CW, Mackenbach JP, Huisman M, van Oyen H, Deboosere P, Gadeyne S, Kunst AE. The contribution of specific diseases to educational disparities in disability-free life expectancy. Am J Public Health. 2005; 95(11): 2035-41

41.Bronnum-Hansen H, Juel K. Impact of smoking on the social gradient in health expectancy in Denmark. J Epidemiol Community Health. 2004; 58(7): 604-10

42. Instituto Nacional de Estadística. Anexo 3. Lista reducidas de causas de defunción. En: Manual de causas de defunción (CIE-10). Versión 7.1. Madrid, INE; $2007 . \mathrm{p} . \quad 1-4 . \quad$ Disponible en : www.ine.es/daco/daco42/sanitarias/listas_02.doc 